\title{
SINGLE TREE THREE-DIMENSIONAL MODEL CONSTRUCTION AND IMPACT FACTOR EXTRACTION BASED ON OBLIQUE PHOTOGRAMMETRY
}

\author{
Wenfei $\mathrm{XI}^{1 *}$, Dongsheng $\mathrm{LI}^{2}$ and Zilong $\mathrm{ZHAO}^{3}$
}

${ }^{1}$ College of Tourism and Geographic Sciences, Yunnan Normal University, Kunming 650500, Yunnan, China

${ }^{2}$ Kunming Metallurgy College. Kunming 650033, Yunnan, China

${ }^{3}$ Yunnan Haiju Geographic Information Technology Co.,Ltd, Kunming 650000, Yunnan, China

* Autor de correspondencia: xiwenfei911@163.com

(Received May 2018; accepted July 2018)

Key words: unmanned aerial vehicles (UAV), oblique photogrammetry, three-dimensional structure model, 3D laser scanning, point cloud data

\begin{abstract}
Based on the digital photogrammetry principle and the computer vision theory, this paper utilizes Unmanned Aerial Vehicle (UAV) oblique photogrammetry technology to construct vegetation canopy structure three-dimensional model and to extract threedimensional model impact factor by conducting image data orientation, matching and aerial triangulation. In order to verify whether the extraction factor is correct, this paper adopts vegetation point cloud data which are obtained from three-dimensional laser scanning. Combined with the actual case analysis, the results of the analysis show that the factor data extracted from the model are reliable.
\end{abstract}

Palabras clave: vehículo aéreo autónomo, fotogrametría oblicua, modelo de estructura tridimensional, escaneo láser 3D, nubes de puntos

\section{RESUMEN}

Con base en el principio de fotogrametría digital y la teoría de visión computacional, este trabajo utiliza la tecnología de fotogrametría oblicua con vehículo aéreo autónomo para construir un modelo tridimensional de la estructura del dosel vegetal y para extraer un modelo tridimensional de factor de impacto por medio de orientación de imágenes de datos y acoplamiento y triangulación aéreas. Para verificar si el factor de extracción es correcto, se adopta la nube de puntos de vegetación que se obtiene del escaneo láser tridimensional. Combinados con un caso real de análisis, los resultados demuestran que el factor extraído de los datos del modelo es confiable.

\section{INTRODUCTION}

With the development of virtual reality, computer graphics, geographic information system subject and computer performance, many scholars have begun to pay attention to the three-dimensional geometric model of trees to simulate the growth process, and used the model to describe various characteristic 
variables, such as, tree height, diameter, and crown, etc (Cheng et al. 2005, Liyu et al. 2006, Allen et al. 2005, Bene and Mill 2002, Lintermann and Deussen 1999, Xi et al. 2017). According to the characteristic variables, different scales biophysical processes are described (Omasa et al. 2007, Rosell et al. 2009). In addition, plant model structure and function can help to understand the growth process and environment impact, such as how interspecific competition affects the three-dimensional shapes of the canopy (Allen et al. 2005, Sievanen et al. 2009, Seidel et al. 2011). Due to the large variety of trees with different shapes in nature, many scholars are studying how to measure and obtain the geometry information effectively (Hongyu et al. 2013).

At present, there are 3 main existing trees 3D modeling methods and they are the methods which are based on growth rules modeling method, image modeling method and sketch modeling method. Each possesses its own advantages, disadvantages and applicability (Ding et al. 2011). The method based on growth rules determines plants three-dimensional shape by defining and adjusting geometric parameters which mainly includes L-system and its derivative system, etc (Prusinkiewicz and Lindenmayer, 1990). The method based on image modeling utilizes the automatic extraction of plant characteristics from several images of a single or different perspective to build a model. The main scholars are Shlyakhter, Quan, Tan and Teng (Shlyakhter et al. 2001, Quan et al. 2006, Tan et al. 2007, Teng and Chen, 2009). The method based on sketch utilizes plant growth rule or existing trees model library to generate 3D models. The main scholars are Okabe, Onishi, etc (Okabe et al. 2005, Onishi et al. 2006). In addition to the methods described above, the relevant mathematical method can also refer to (Gao et al. 2016, Gao and Wang 2016a, Gao and Wang 2016b).

Recently, unmanned aerial vehicles (UAV) technology has attracted much attention from public. It becomes more and more heated to study on modeling by means of photogrammetry theory. Unmanned aerial vehicle (UAV) is a high-tech product that relies on multiple technologies and hardware. Using unmanned aerial vehicles (UAV) can collect target object information comprehensively and take a set of digital images at different angles around the measured object. It can obtain rich texture information, which is suitable for small-scale, low-cost, low-precision measurement task. For instance, the unmanned aerial vehicle (UAV) are used by Chai and others to conduct landscape DEM establishment (Chai et al. 2015, Xi and Li 2017).
This paper takes a tree in the campus of Yunnan normal university as an example. The unmanned aerial vehicle (UAV) photography technology and 3D laser scanner are used to collect the data and the UAV image processing software is used to build 3D model. It extracts the impacted factors are extracted from the constructed model, and then validated by the 3D laser scanning data. SIFT algorithm is used in following experiment which is described as follow.

\section{MATERIAL AND METHODS}

\section{Sift algorithm principle}

\section{The establishment of Gaussian differential pyramid}

The SIFT algorithm, proposed by David G. Lowe, is a feature-based matching algorithm based on scale space, mainly using Gaussian differential pyramid model (David and Owe 2004). The Gaussian pyramid is essentially a multi-scale representation of the signal, Gaussian blurring the same signal or picture many times, and down-sampling to produce multiple sets of signals or pictures at different scales for subsequent processing. On the basis of a series of reasonable assumptions, Koenderink and Lindeberg proved that the Gaussian function probably is the only spatial kernel scale to realize image scale conversion. One tile of image spatial scale can be defined as:

$L(x, y, s)=G(x, y, s) * I(x, y)$

where * represents the sum of convolution operation, $(x, y)$ denotes the space coordinates of pixel, $s$ is scale coordinates of pixel, and $G(x, y, s)$ is scale variable Gaussian kernel function, which is defined as:

$G(x, y, s)=\frac{1}{2 p s^{2}} e^{-\left(x^{2}+y^{2}\right) / 2 s^{2}}$

David G. Lowe raised that it uses Gaussian difference operator to establish image Gaussian difference scale space. It is able to obtain Gaussian difference images $\mathrm{D}(x, y, s)$ with different scales by using the Gaussian Difference Kernels and Image Convolution on Different Scales. All Gaussian difference images together make up the Gaussian difference pyramid of the image.

Where

$$
\begin{aligned}
& \mathrm{D}(x, y, s)=(G(x, y, k s)-G(x, y, s)) * \\
& \quad * I(x, y)=L(x, y, k s)-L(x, y, s)
\end{aligned}
$$

Construction process of image Gaussian difference pyramid is shown in the left part of Fig.1. In the group, the images from the upper layer are convo- 


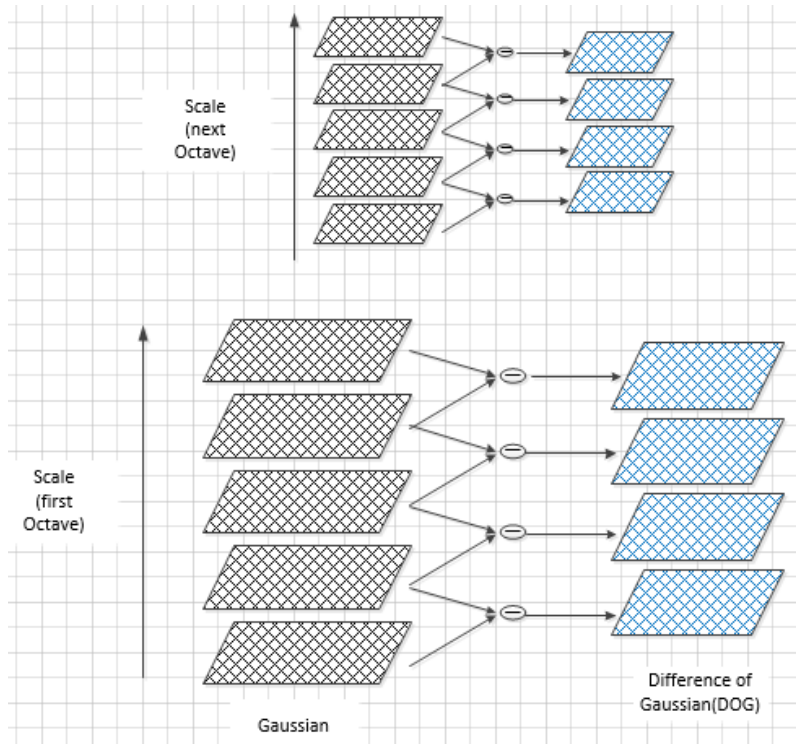

Fig. 1. The generation of Gaussian difference pyramid

luted with the Gaussian functions of different scales to obtain the spatial images in which the constant scale factor is increased $\mathrm{k}$ times. In other words, the scale of first layer is s, the scale of second layer is $\mathrm{ks}$, and the next layer follows this rule. The image of the first layer of each group is obtained from the first layer sample of the previous set of images. The size of the image after sampling is $1 / 4$ of the size of the images of the previous group, but scale size is twice of the original one. In this way, the Gaussian difference pyramid is constructed.

The right part of Fig.1 describes the process how to construct Gaussian difference pyramid. In the group, Gaussian difference image is obtained after the subtraction between adjacent images. Then, after repeating the previous operations on the full set of images, we get the Gaussian difference pyramid.

\section{Generation of feature point descriptor}

After the key point SIFT feature area is generated, firstly the axis direction is rotated to the main direction of the key points, which ensures the rotation invariance and then we select the neighborhood window with the key as the center, as shown in the left part of Fig.2.

The central pixel is the key point to be described. The surrounding little grids represent the pixels of the neighborhood key points; the length of the arrow represents the gradient value of the pixel and the direction of the arrow represents the gradient direction of the pixel. The circles in the figure represent the range of Gaussian weighting of pixel values (Rai et al. 2018). As shown in right part of Fig.2. In every sub block, if we calculate the gradient direction histogram in eight directions, we can get a seed point. When all the seed points are obtained, we get a descriptor.

\section{Data acquisition}

This paper takes a tree in the campus of Yunnan normal university as an example and the oblique photogrammetry method is used to circumnavigate the target and take pictures for one week. The unmanned aerial vehicles (UAV) chosen in our experiment is the Da jiang PHANTOM3 which uses GPS/GLONASS dual-mode position system with 12.4 million pixels. Smart3dCapture4.0 software is chosen as our modeling software. Scanstation C10 is used for point cloud data acquisition. There are two points to collect data, and the scanned point cloud data are denoised and spliced in the end.

The main idea of this experiment is designed as follows:

1. Using unmanned aerial vehicle (UAV) to obtain the original image data. In this process, the overlap degree of the obtained images should be not less than $70 \%$. The uniform source of light should be ensured in the field data collection.

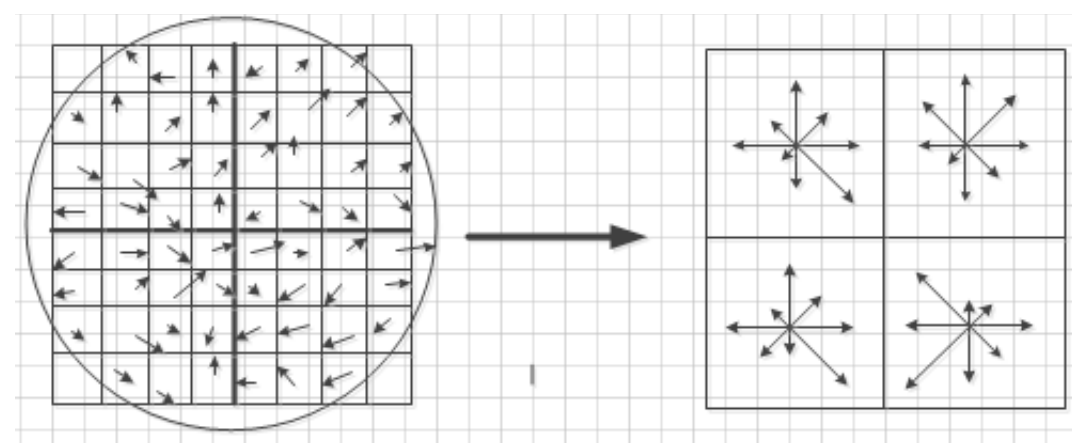

Fig. 2. Feature vector generated by critical point neighborhood gradient information 


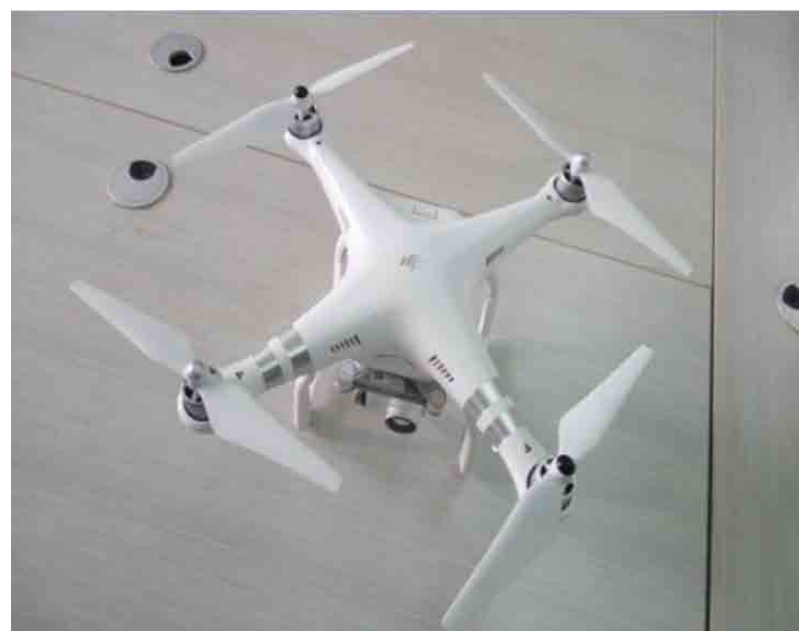

Fig. 3. Unmanned aerial vehicles

2. The quality of the acquired image data must be checked. The software is used to check whether the image meets the quality requirements of modeling. If not, the UAV must fly again to collect enough data.

3. Smart3d Capture4. 0 is used to conduct $3 \mathrm{D}$ modeling;

4. 3D laser scanner is used to acquire data;

5. Data comparative analysis.

The main operating process is shown in Fig.4

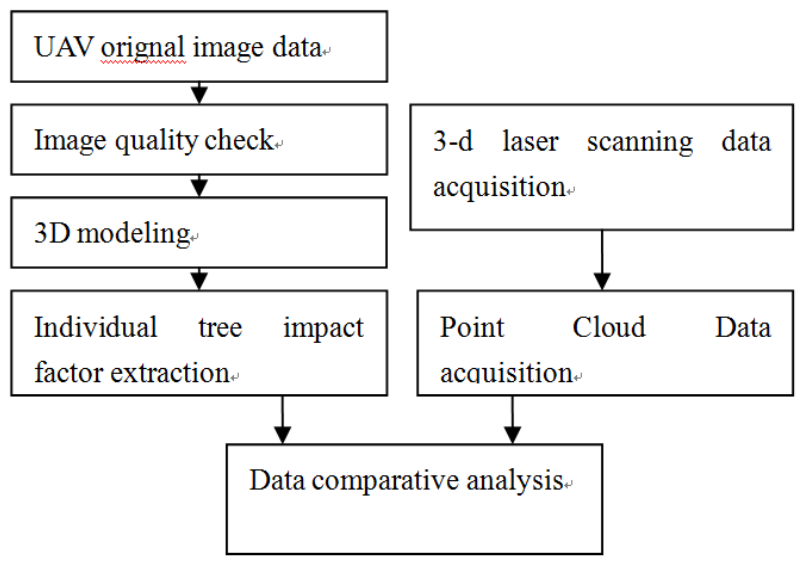

Fig. 4. Data processing flow of image

\section{RESULTS AND DISCUSSION}

In accordance with the above operation process, unmanned aerial vehicle (UAV) is used to collect the pictures of an individual tree and the target image is shown in Fig.5. The flight height of the unmanned aerial vehicle (UAV) is 50 meters and 235 pieces of images are obtained. The degree of the picture overlap is $85 \%$. After data quality inspection and rectification of them, it constructs $3 \mathrm{D}$ model with the Smart3dCapture 4.0 is used to construct 3D models and finally the space triangulation is obtained, which is shown in Fig.6.

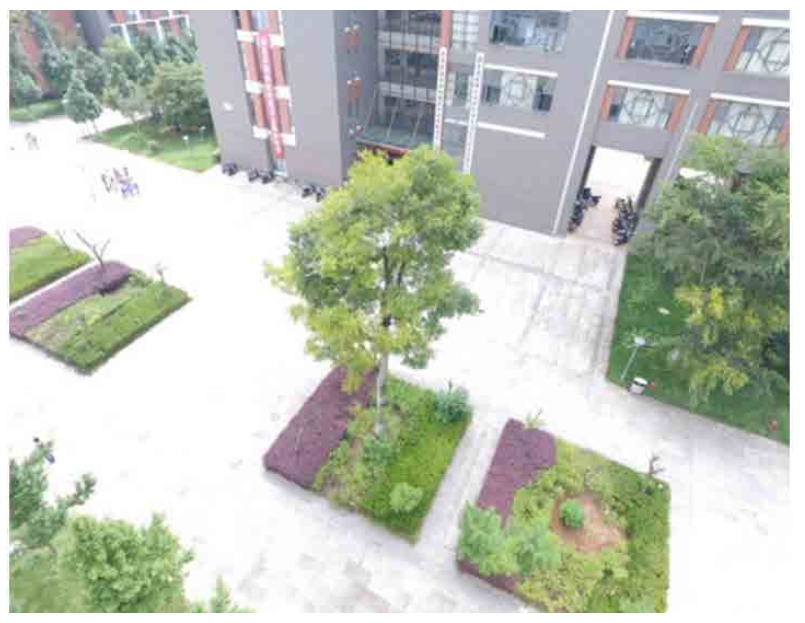

Fig. 5. Target image

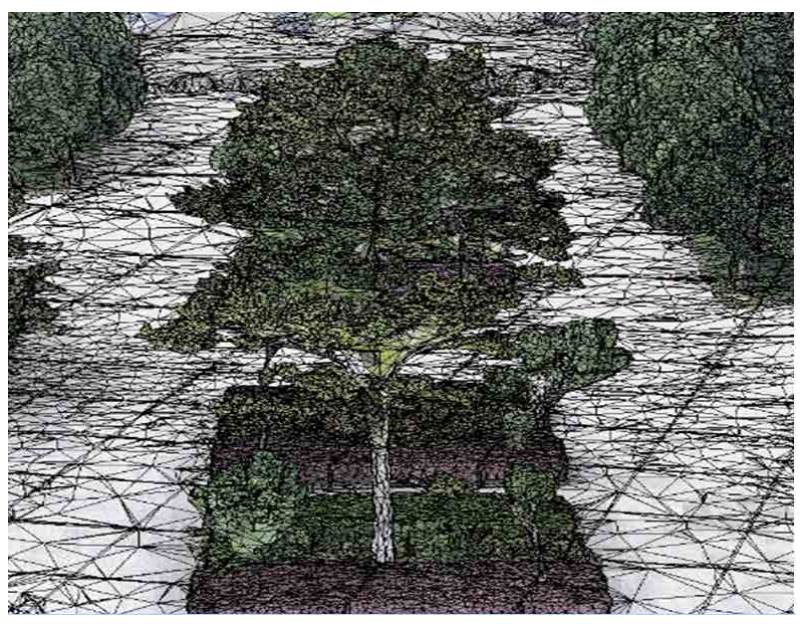

Fig. 6. Canopy space triangulation

The three-dimensional model is constructed by using the constructed triangulated space. The 3D model is shown in Fig.7. It shows that the hierarchy of canopy 3D model is much clearer than the original picture. As a result, the model constructed by oblique 
photogrammetry can reflect the structural information of the target object well.

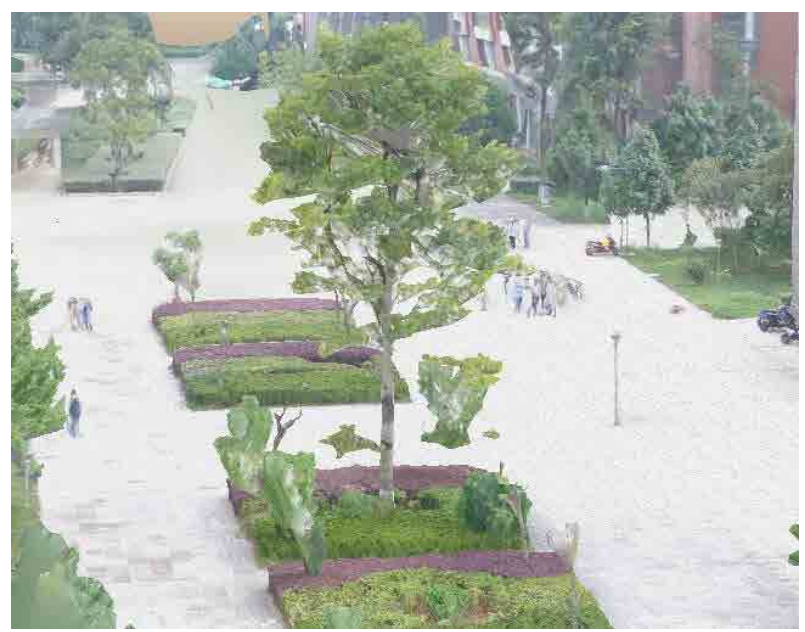

Fig. 7. Canopy 3D model

The result of target objects 3D model that obtained from the laser scanner is shown in Fig. 8. Ranging accuracy is $1.2 \mathrm{~mm}+10 \mathrm{pmm}$, and the acquired point cloud data is shown in Fig 9.

The point cloud data are obtained by 3D laser scanner and the scanned point cloud data was stitched

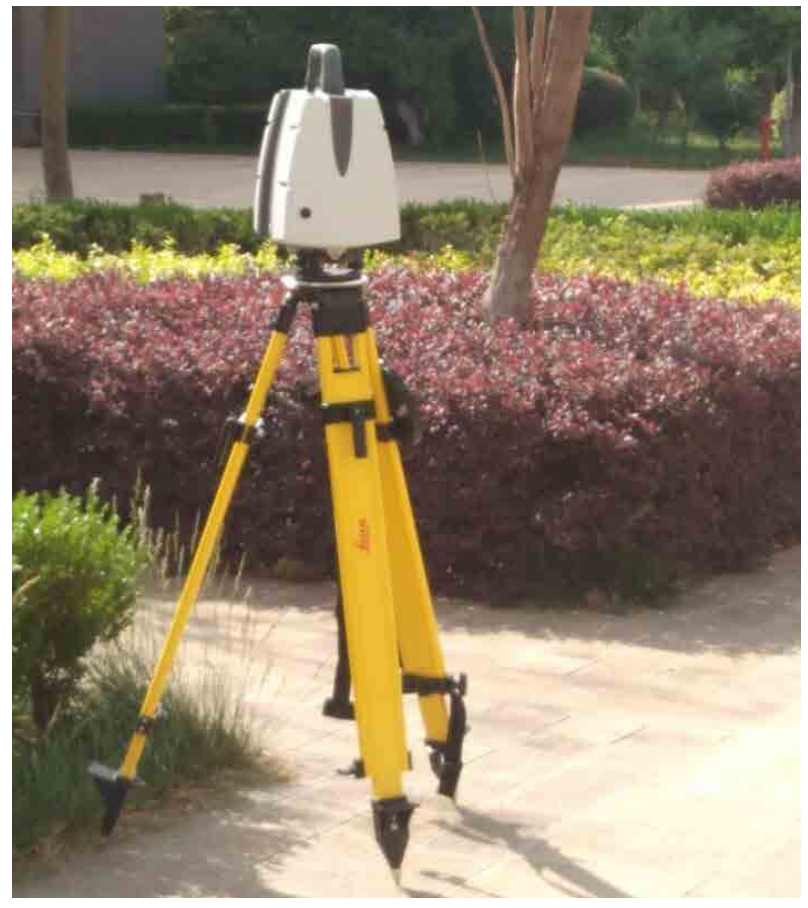

Fig. 8. Data collection

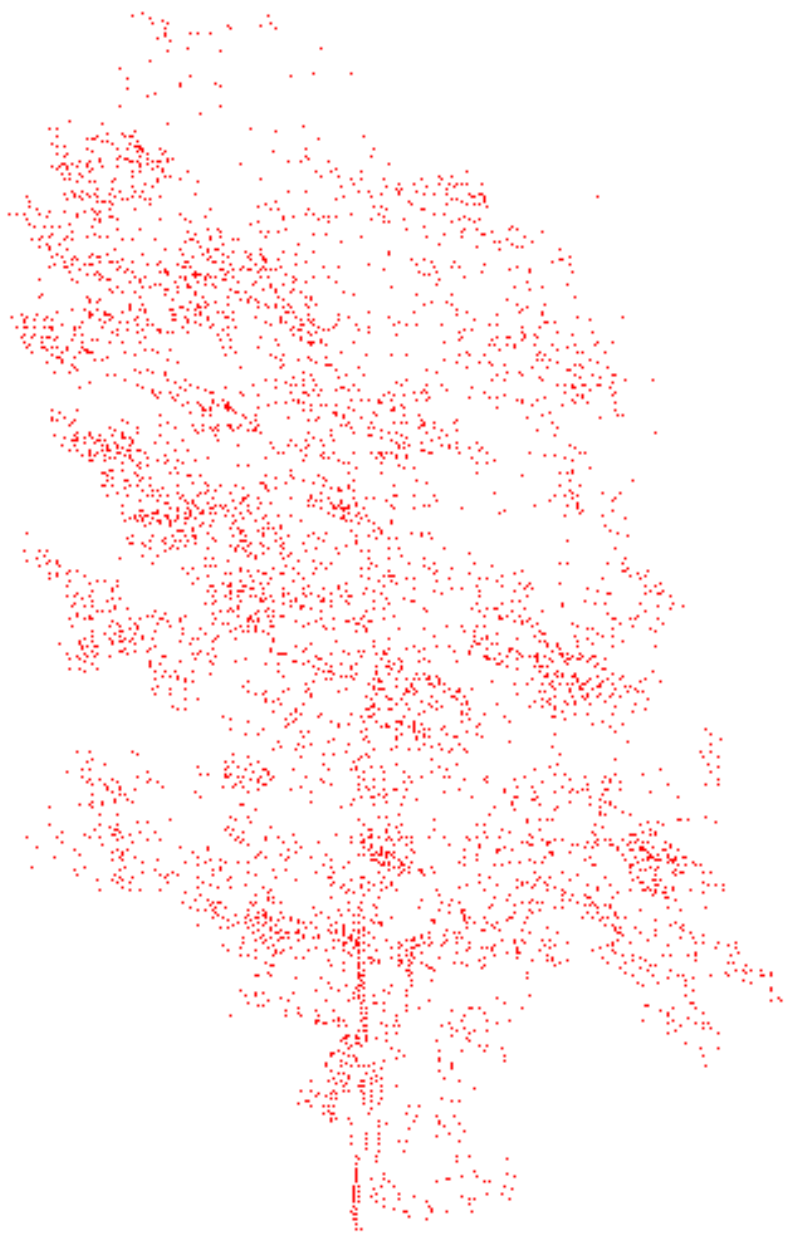

Fig. 9. Target point cloud data

by the target. Then we get the independent coordinate system. It can be seen from the point cloud data that both the vegetation Outline and structure are clear.

Analyzing the three-dimensional model obtained by the UAV and the point cloud data got from the three-dimensional laser scanner, it can be seen from Table. 1 that the factor extracted by oblique photogrammetry is close to the data obtained by $3 \mathrm{D}$ laser scanner, which basically meets the accuracy requirements of factor extraction.

TABLE I. DATA COMPARISON ANALYSIS

\begin{tabular}{lcc}
\hline & $\begin{array}{c}\text { Tree } \\
\text { height }\end{array}$ & $\begin{array}{c}\text { The maximum diameter } \\
\text { of the tree }\end{array}$ \\
\hline $\begin{array}{l}\text { Factor extracted } \\
\text { by (UAV) model ion }\end{array}$ & $14.89 \mathrm{~m}$ & $44.5 \mathrm{~cm}$ \\
3D laser scanner & $14.29 \mathrm{~m}$ & $36.4 \mathrm{~cm}$ \\
Error & $0.6 \mathrm{~m}$ & $8.1 \mathrm{~cm}$ \\
\hline
\end{tabular}




\section{CONCLUSION}

In this paper, we use unmanned aerial vehicle (UAV) image oblique photogrammetry to construct 3D modeling from which the vegetation factors are obtained. The results of extraction show to be close to the data obtained by $3 \mathrm{D}$ laser scanner. It proves that the method is useful to extract vegetation factors of an individual tree. Project to the future, the method need to be further studied its feasibility in a larger density forest region.

\section{ACKNOWLEDGMENTS}

We thank the reviewers for their constructive comments on improving the quality of this paper. This research was supported in part by the Yunnan Provincial Department of education research foundation (2016ZZX067). Yunnan provincial science and technology department fund (2017FB078).

\section{REFERENCES}

Allen M., Prusinkiewicz P., and DeJong T. (2005). Using L-systems for modeling source-sink interactions, architecture and physiology of growing trees: The L-PEACH model. New Phytologist. 166 (3), 869-880.

Bene B., and Milln E.U. (2002). Virtual climbing plants competing for space. Proceeding of the Computer Animation IEEE Computer Society, 33-43.

Chongcheng C., Liyu T., Bing Q., Jianwei L., and Song S. (2005). Construction of information managementbased virtual forest landscape and its application. Chinese Journal of Applied Ecology, 16 (11), 2047-2052.

David G.I., and Owe. (2004). Distinctive Image Features from Scale-Invariant Key points . International Journal of Computer Vision. 60 (2), 91-110.

Ding L., Cheng C.C., Yu T.L., Jie Z., and Min W.Q. (2011). $3 \mathrm{D}$ tree modeling based on the parametric curve and its integral. Journal of Fuzhou University (Natural Science Edition), 39 (3), 367-374.

Gao W., and Wang W. (2016b). The eccentric connectivity polynomial of two classes of nanotubes, In press. Chaos, Solitons and Fractals, 89, 290-294.

Gao W., and Wang, W. (2016a). The fifth geometric arithmetic index of bridge graph and carbon nanocones. Journal of Difference Equations and Applications.

Gao W., Guo Y., Wang K. (2016). Ontology algorithm using singular value decomposition and applied in multidisciplinary. Cluster Computing the Journal of
Networks Software Tools and Applications, 19 (4), 2201-2210.

Hongyu H., Chongcheng C., Jie Z., and Ding L. (2013). Tree Geometrical 3D Modeling from Terrestrial Laser Scanned Point Clouds: A Review. Scientia Silvae Sinicae, 49 (4). 123-130.

Lintermann B, Deussen O. (1999). Interactive modeling of plants. IEEE Computer Graphics and Applications, 19 (1), 56 -65.

Liyu T., Chongcheng C., and Bing Q. (2006). Advances in Forest Landscape's Computer Modeling and Visualization. Scientia silvae sinicae, 42 (10), 109-116.

Okabe M., Owada S., and Igarash T. (2005). Interactive design of botanical trees using freeh and sketches and example-based editing. Proc of Eurographics, 487-496.

Omasa K., Hosoi F., and Konishi A. (2007). 3D lidar imaging for detecting and understanding plant responses and canopy structure. Journal of Experimental Botany. 58 (4), $881-898$.

Onishi K., Murakami N., Kitamura Y., and Kishino F. (2006). Modeling of trees with interactiveL-system and 3D gestures. Biologically Inspired Approaches to Advanced Information Technology, 3853, 222-235.

Prusinkiewicz P., and Lindenmayer A. (1990). The algorithmic beauty of plants. Springer-Verlag, Berlin, Germany.

Quan L., Tan P., Zeng G., Yuan L., Wang J., and Kang S.B. (2006). Image-based plant modeling. TCM Transactions on Graphics (TOG). Proceeding of acm siggraph 25 (3), 599-604.

Rosell J.R., Llorens J., Sanz R., Arnó J., Dasi M.R., Masip J., Escolà A., Camp F., Solanelles F., Gràcia F., Gil E., Val L., Planas S., and Palacín J. (2009). Obtaining the three-dimensional structure of tree orchards from remote 2D terrestrial LIDAR scanning. Agricultural and Forest Meteorology, 149 (9), 1505-1515.

Seidel D., Leuschner C., Müller A., and Krause B. (2011). Crown plasticity in mixed forests Quantifying asymmetry as a measure of competition using terrestrial laser scanning. Forest Ecology and Management, 261 (11), 2123-2132.

Shlyakhter I., Rozenoer M., Dorsey J., and Teller S. (2001). Reconstructing 3D tree models from instrumented photographs. IEEE Computer Graphics and Applications, 21 (3), $53-61$.

Sievanen R., Perttunen J., Nikinmaa E., and Posada J.M. (2009). Invited talk:functional structural plant modelscase LIGNUM. Proc of Plant Growth Modeling and Applications 09, Los Alamitos, CA, USA, IEEE Computer Society, 3-9.

Tan P., Zeng G., Wang J., Kang S.B., and Quan L. (2007). Image-based tree modeling. ACM, Transation on Graphics, 26 (3), 87-88. 
Teng C.H., and Chen Y.S. (2009). Image-based tree modeling from a few images with very narrow viewing range. The Visual Computer, 25 (4), 297-307.

Wenfei X., and Dongsheng L. (2017). Steep Slope DEM Model Construction based on the Unmanned Aerial Vehicle (UAV) Images. Sains Malaysiana, 11, 21192124.
Wenfei X., Zhengtao S., and Dongsheng L. (2017). Comparisons of feature extraction algorithm based on unmanned aerial vehicle image. Open Phys. 15, 472-478.

ZiWei C., Jun K., Li W., Xin Z., Hailang Q. (2015). The Construction of DEM in Mountain Plantation Landscape based on uav images. Remote Sensing Technology and Application, 30 (3), 504-508. 